\title{
Produção de clones de taro em função dos tipos de mudas
}

\author{
Néstor A. Heredia Zárate ${ }^{1}$; Maria do Carmo Vieira ${ }^{1}$ \\ UFMS-DCA, C. Postal 533, 79804-970 Dourados-MS; E-mail: nheredia@ceud.ufms.br; 'Bolsista de Produtividade em Pesquisa do CNPq.
}

\begin{abstract}
RESUMO
Procurou-se conhecer as produções de massas frescas das plantas, visando maior retorno econômico, de dois clones de taro (Chinês e Macaquinho) propagados por quatro tipos de mudas [rizomafilho pequeno (RFP), metade de RFP, rizoma-filho grande (RFG) e metade de RFG], arranjados como fatorial $2 \times 4$, no delineamento experimental de blocos casualizados, com quatro repetições. $\mathrm{O}$ experimento foi realizado em Dourados, entre 13/9/00 e 11/5/01, em parcelas de 1,50 x 1,98 m, com 24 plantas em fileira dupla. A colheita foi realizada aos 240 dias após o plantio, quando foram avaliadas as produções totais de massas frescas de rizomas-mães (RM) e de rizomas-filhos $(\mathrm{RF})$, incluindo as produções das classes grande, média e pequena. Avaliou-se os custos das mudas e a renda bruta esperada. A produção total de RF variou significativamente entre clones. As produções de $R M, R F$, total de rizomas $(R M+R F)$ e de rizomas comerciais (RF das classes grande + média) do clone Macaquinho foram as maiores, com diferenças de 1,$84 ; 7,11 ; 8,95$ e 4,50 t ha-1, em relação às do 'Chinês', com aumento da renda bruta (rizomas comerciais x preço pago ao agricultor) calculada em $\mathrm{R} \$ 841 \mathrm{ha}^{-1}$. As maiores produções de rizomas $\left(46,44 \mathrm{t} \mathrm{ha}^{-1}\right)$ e de rizomas comerciais $\left(27,93 \mathrm{t} \mathrm{ha}^{-1}\right)$ e a maior renda bruta calculada ( $\left.\mathrm{R} \$ 5.586\right)$ foram obtidas no clone Macaquinho, propagado com metade de RFP.
\end{abstract}

\begin{abstract}
Yield of taro clones as a function of cutting types

Fresh mass yield of plants of two taro clones (Chinês and Macaquinho) was evaluated to obtain a better gross income for producers in Dourados, Brazil. The taro clones were propagated through four cutting types [small cormel (RFP), half RFP, big cormel (RFG) and half RFG], arranged as a $2 \times 4$ factorial scheme in a randomized block design with four replications, between 13/09/2000 and $11 / 05 / 2001$. Plot measured $1.50 \times 1.98 \mathrm{~m}$, with 24 plants arranged in double rows. Plants were harvested 240 days after planting date, when total yield of fresh mass of corms (RM) and of cormels (RF) was evaluated, classified as big, medium or small sized. Cutting costs and expected gross income also were obtained. The yield of RM, $\mathrm{RF}$, total of rhizomes $(\mathrm{RM}+\mathrm{RF})$ and commercial rhizomes (RF of big + medium classes) of 'Macaquinho' taro were higher than 'Chinês' with differences of $1.84 ; 7.11 ; 8.95$ and $4.50 \mathrm{t} \mathrm{ha}^{-1}$ and an increase of gross income (commercial rhizomes $\mathrm{x}$ price paid to farmer) evaluated in $\mathrm{R} \$ 841 \mathrm{ha}^{-1}$. The highest total $\left(46.44 \mathrm{tha}^{-1}\right)$ and commercial $\left(27.93 \mathrm{t} \mathrm{ha}^{-1}\right)$ yield of rhizomes and evaluated gross income $(\mathrm{R} \$ 5,586)$ were obtained with Macaquinho clone, propagated with half RFP.
\end{abstract}

Keywords: Colocasia esculenta, propagation, yield, income.

Palavras-chave: Colocasia esculenta, propagação, produtividade, retorno econômico.

\section{(Recebido para publicação em 9 de setembro de 2002 e aceito em 4 de julho de 2003)}

$\mathrm{O}$ taro (Colocasia esculenta (L.) Schott) é uma Araceae utilizada na indústria e dieta alimentar de muitos países tropicais, por ser alimento rico em amido, ter grande produção por unidade de área, ser pouco exigente em gastos com mão-de-obra e insumos e por ser de fácil conservação (Abramo,1990). As plantas de taro têm grande faixa de adaptação climática e de solos. Por isso podem sobreviver a condições consideradas adversas para outras culturas, crescendo em locais com excesso de água, à sombra ou com períodos de seca, o que possibilita o uso de áreas impróprias para a agricultura tradicional. O tempo requerido para alcançar a maturidade e produzir rizomas varia de acordo com a disponibilidade de água e de luz e, especialmente, temperatura (Heredia Zárate, 1990a). Contudo, a produtividade é variável por causa do desconhecimento das características genotípicas das diferentes cultivares e pelas diferen- ças nas práticas de plantio e de irrigação (Wang, 1983, Heredia Zárate, 1988).

No Hawaí, o número de variedades ou clones de taro chega a cem, mas apenas cinco ou seis são comerciais (Wang, 1983). No Brasil, nas regiões do Rio Doce-MG (Santos et al., 1983), no Município de Inhapim-MG (Santos, 1994) e na região de Juiz de Fora-MG (Fonseca, 1994), são cultivados geralmente os clones Japonês, Chinês e Macaquinho. Em Mato Grosso do Sul, estão sendo estudados e incentivados o cultivo dos clones Japonês, Branco, Macaquinho, Chinês e Cem por Um, tanto nas áreas de várzea seca do pantanal (Heredia Zárate, 1995) como em solos irrigados e mantidos "sempre úmidos" (Heredia Zárate \& Yamaguti, 1994; Heredia Zárate, 1995; Heredia Zárate et al., 2000).

Para espécies propagadas vegetativamente, o tamanho da muda é fator conflitante. Quanto ao taro, pode ser propagado por rizomas centrais ou "rizomas-mães", rizomas chupões ou "rizomas-filhos"; por meio de mudas (rizomas cuja parte basal tenha sido cortada, ficando com cerca de $6,0 \mathrm{~cm}$ de comprimento, além do corte da parte aérea para ficar com 15,0 a 25,0 cm de pecíolo). Outras opções consistem em plantar os "rizomas-filhos" pequenos com folhas jovens e utilizar os rizomasmães ou rizomas-filhos, inteiros ou cortados (Heredia Zárate, 1990b). Casos parecidos são observados na multiplicação da mandioquinha-salsa (Arracacia xanthorrhiza), da qual utilizam-se rebentos ou mudas (Vieira, 1995; Sediyama \& Casali, 1997) e do inhame, do qual se utilizam os rizomas e/ou tubérculos como em Dioscorea alata (Heredia Zárate et al., 1998) e as túberas-semente inteiras ou partes das "cabeças", meio e ponta, no inhame D. cayennensis (Santos, 1996; Oliveira et al., 2000). 
Produção de clones de taro em função dos tipos de mudas

Tabela 1. Produção de massa fresca de rizomas, mães e filhos, de dois clones de taro. Dourados, UFMS, 2000-2001.

\begin{tabular}{lccccc}
\hline \multirow{2}{*}{ Clones } & \multicolumn{5}{c}{ Produção de rizomas (t ha ${ }^{-1}$ ) } \\
\cline { 2 - 6 } & \multirow{2}{*}{ Mães } & Total & Grande & Médio & Pequeno \\
\cline { 2 - 6 } & & 26,08 a & 9,34 & 11,43 & 5,31 \\
\hline Chinês & 5,38 & 33,19 b & 11,44 & 13,83 & 7,92 \\
Macaquinho & 7,22 & 29,63 & 32,16 & 37,40 & 46,07 \\
\hline C.V. $(\%)$ & 58,48 & &
\end{tabular}

Médias seguidas por letras diferentes, nas colunas, diferem entre si pelo teste $\mathrm{F}$, a $5 \%$ de probabilidade

A qualidade do material de plantio determina diferenças na velocidade de enraizamento, crescimento e, conseqüentemente, produção e extensão do ciclo vegetativo. Vários aspectos das mudas ainda não foram estudados e, se o foram, os resultados ainda não são conclusivos. Em razão disso, muitas informações disponíveis originam-se da experiência de produtores e de extensionistas, além dos poucos pesquisadores que estudam essa espécie. Heredia Zárate et al. (1998) observaram em inhame que o uso de mudas grandes (146,9 g para o ‘Pezão’ e 186,3 g para o 'Flórida') induziu maiores produções de rizomas dos clones Roxo, Caramujo, Pezão e Flórida e que as melhores mudas para o clone Mimoso foram as obtidas do corte das pontas dos rizomas contendo $84,1 \mathrm{~g}$. As produções de tubérculos dos clones Caramujo e Pezão foram maiores com o uso de mudas formadas pelas pontas dos rizomas contendo 71,6 $\mathrm{g}$ e $56,6 \mathrm{~g}$, respectivamente.

A importância de se conhecer bem o tipo, o tamanho e a forma como a muda de taro deve ser plantada está principalmente no fato de o ciclo de cultivo ser de cinco a nove meses e, portanto, há necessidade que se estabeleça a população final desejada (Heredia Zárate, 1990a e b). Este trabalho teve como objetivo conhecer a capacidade produtiva de dois clones de taro, utilizando-se quatro tipos de mudas, visando um maior retorno econômico para o produtor.

\section{MATERIAL E MÉTODOS}

O trabalho foi desenvolvido na Universidade Federal de Mato Grosso do Sul em Dourados, de 13/9/00 a 11/5/01, em Latossolo Vermelho distroférrico, de textura argilosa, com as características:
4,9 de $\mathrm{pH}$ em $\mathrm{CaCl}_{2} ; 34,0 \mathrm{~g} \mathrm{dm}^{-3}$ de M.O; 100,0 e $34,0 \mathrm{mg} \mathrm{dm}^{-3}$ de $\mathrm{P}$ e S, respectivamente, e 2,4; 36,0 e 19,0 $\mathrm{mmol}_{\mathrm{c}} \mathrm{dm}^{-3}$ de $\mathrm{K}, \mathrm{Ca}$ e $\mathrm{Mg}$, respectivamente. $\mathrm{O}$ município de Dourados situa-se em latitude de $22^{\circ} 13^{\prime} 16^{\prime} \mathrm{S}$, longitude de $54^{\circ} 17^{\prime} 01^{\prime \prime} \mathrm{W}$ e altitude de $430 \mathrm{~m}$. O clima da região, segundo a classificação de Köppen é Mesotérmico Úmido, do tipo Cwa, com temperaturas e precipitações médias anuais variando de 20 a $24^{\circ} \mathrm{C}$ e de $1250 \mathrm{~mm}$ a $1500 \mathrm{~mm}$, respectivamente.

Foram estudados dois clones de taro (Chinês e Macaquinho) propagados por quatro tipos de mudas [rizomas-filhos pequenos (RFP), metade de RFP, rizomas-filhos grandes (RFG) e metade de RFG], arranjados como fatorial 2 $\mathrm{x} 4$, no delineamento experimental de blocos casualizados, com quatro repetições. Cada parcela teve $1,50 \mathrm{~m}$ de largura e 1,98 m de comprimento, com uma fileira dupla de plantas, espaçamentos de $0,165 \mathrm{~m}$ entre plantas, $0,60 \mathrm{~m}$ entre fileiras simples e $0,90 \mathrm{~m}$ entre fileiras duplas, perfazendo população de 79.992 plantas ha-1.

O solo da área do experimento foi preparado com aração, gradagem e levantamento de canteiros com rotoencanteirador. No dia do plantio, foram abertos sulcos de aproximadamente $0,10 \mathrm{~m}$ de largura por $0,10 \mathrm{~m}$ de profundidade, onde colocaram-se as mudas em posição deitada. As massas médias das mudas foram de $81,25 \mathrm{~g}$; 41,95 g; 20,30 g e 11,15 g para RFG; metade de RFG, RFP e metade de RFP, respectivamente. As irrigações foram feitas por aspersão, com turnos de rega de três a quatro dias, de forma a manter o solo com umidade em torno de $70 \%$ da capacidade de campo (após observações visuais e no tato). Não foram utili- zados agrotóxicos e as capinas, em número de quatro, foram feitas manualmente com auxílio de enxada.

A colheita foi realizada aos 240 dias após o plantio, quando a maioria das plantas tinham no mínimo $70 \%$ da parte aérea com sintomas de senescência. Foram avaliadas as produções totais de massas frescas de rizomas-mães (RM) e de rizomas-filhos (RF), incluindo as produções das classes (classificação visual) grandes, médias e pequenas. Os dados obtidos foram submetidos à análise de variância e quando verificou-se significância pelo teste $\mathrm{F}$, aplicou-se o teste de Tukey, a 5\% de probabilidade. Também foram calculados os custos das mudas e a renda bruta esperada.

\section{RESULTADOS E DISCUSSÃO}

As maiores produções de matéria fresca de RM e de RF foram do taro Macaquinho, com diferenças de 1,84 e $7,11 \mathrm{t} \mathrm{ha}^{-1}$, respectivamente, em relação ao 'Chinês' (Tabela 1). Esse fato permite supor que a partição dos fotoassimilados é função do genótipo utilizado e das relações fonte-dreno (Larcher, 2000) e que os RM já tinham alcançado a maturidade e o máximo crescimento, com provável aumento da translocação dos fotossintatos das folhas diretamente para os RM e destes para os RF (Hashad et al.,1956). Por isso, encontraram-se aumentos de $8,95 \mathrm{t} \mathrm{ha}^{-1}$ do total de rizomas e de 4,50 tha ${ }^{-1}$ de rizomas comerciais para o clone Macaquinho, em relação ao 'Chinês', com aumento da renda bruta de R $\$ 841$ ha $^{-1}$.

Quando relacionadas as produções de massa fresca de RM e de RF (Tabela 2) e as produções totais de rizomas e de rizomas comerciais, os resultados obtidos mostraram que para a propagação 
Tabela 2. Produção de massa fresca de rizomas (mães e filhos) de dois clones de taro em função de quatro tipos de mudas. Dourados, UFMS, 2000-2001.

\begin{tabular}{|c|c|c|c|c|c|c|}
\hline \multirow{3}{*}{ Clones } & \multirow{3}{*}{$\begin{array}{c}\text { Tipos de } \\
\text { mudas }\end{array}$} & \multicolumn{5}{|c|}{ Produção de rizomas ( $\left.\mathrm{t} \mathrm{ha}{ }^{-1}\right)$} \\
\hline & & \multirow{2}{*}{ Mães } & \multicolumn{4}{|c|}{ Filhos } \\
\hline & & & Total & Grande & Médio & Pequeno \\
\hline \multirow{4}{*}{ Chinês } & Metade RFP & 5,59 & $31,62 \mathrm{a}$ & $13,43 \mathrm{a}$ & 13,23 & 4,96 \\
\hline & $\mathrm{RFP}^{1}$ & 7,04 & $28,66 \mathrm{ab}$ & $9,68 a b$ & 13,32 & 5,66 \\
\hline & Metade RFG & 4,36 & $19,86 \mathrm{~b}$ & $6,58 \mathrm{~b}$ & 7,62 & 5,66 \\
\hline & $\mathrm{RFG}^{2}$ & 4,54 & $24,18 a b$ & $7,68 a b$ & 11,56 & 4,94 \\
\hline \multirow{4}{*}{ Macaquinho } & Metade RFP & 8,08 & 38,36 & 11,84 & 16,09 & 10,43 \\
\hline & $\mathrm{RFP}^{1}$ & 7,45 & 31,97 & 9,22 & 14,45 & 8,30 \\
\hline & Metade RFG & 7,27 & 32,57 & 13,53 & 11,51 & 7,53 \\
\hline & $\mathrm{RFG}^{2}$ & 6,09 & 29,88 & 11,19 & 13,28 & 5,41 \\
\hline C.V. $(\%)$ & & 58,48 & 29,20 & 32,16 & 37,40 & 46,07 \\
\hline
\end{tabular}

${ }^{1}$ Rizoma-filho pequeno ${ }^{2}$ Rizoma-filho grande

Médias seguidas por letras diferentes, nas colunas, diferem entre si pelo teste de Turkey, a 5\% de probabilidade

dos dois clones foi melhor o uso de mudas formadas por metade de RFP. Deduz-se então que essas mudas, nas fases iniciais de cultivo, podem ter induzido menor crescimento e desenvolvimento das plantas; no entanto, na fase de crescimento dos rizomas tiveram maior quantidade de fotoassimilados a serem translocados para os RM, e os excedentes translocados para os RF (Hashad et al.,1956). Assim, observaram-se diferenças de 12,99 e 12,46 $\mathrm{tha}^{-1}$, entre as maiores e as menores produções total e de rizomas comerciais dentro do clone Chinês, propagado com metade de RFP e metade de RFG, respectivamente e de 10,47 e 4,26 $\mathrm{t} \mathrm{ha}^{-1}$ dentro do clone Macaquinho, propagado com metade de RFP e RFP, respectivamente. Esses resultados mostram relação com o exposto por Larcher (2000), de que o padrão de resposta de uma planta e seu específico potencial de adaptação é característica geneticamente determinada.

As maiores rendas brutas calculadas foram obtidas nas plantas provenientes de metade de RFP, com diferenças de $\mathrm{R} \$ 2.492$ e de R\$ 852 em relação às plantas provenientes de metade de RFG no taro Chinês e de RFP no Macaquinho, respectivamente.

\section{AGRADECIMENTOS}

Ao CNPq, pelas bolsas concedidas, e à FUNDECT, pelos recursos financeiros.

\section{LITERATURA CITADA}

ABRAMO, M.A. Taioba, cará e inhame: o grande potencial inexplorado. São Paulo: Ícone, 1990.80 p. FONSECA, P.C. Sistema de produção de inhame na região de Juiz de Fora-MG. In: ENCONTRO NACIONAL SOBRE A CULTURA DO INHAME, 1, Viçosa. 1987. Anais.... Viçosa: UFV, 1994. p. 53-54.

HASHAD, M.N.; STINO, K.R.; EL-HINNAMY, S.I. Transformation and translocation of carbohidrates in taro plants during growth. Annals of Agricultural Sciences, v. 1, n. 1, p. 261-267, 1956. HEREDIA ZÁRATE, N.A. Curvas de crescimento de inhame (Colocasia esculenta (L.) Schott), considerando cinco populações, em solo seco e alagado. Viçosa, 1988. 95 p. (Tese doutorado), UFV. HEREDIAZÁRATE, N.A. Curvas de crescimento de inhame e da variação na composição química e na umidade do solo, considerando cinco populações e cinco épocas de preparo do solo. In: ENCONTRO NACIONAL SOBRE A CULTURA DO INHAME, 2. Dourados, 1989. Anais.... Campo Grande: UFMS, 1990a. p. 11-42.

HEREDIA ZÁRATE, N.A. Propagação e tratos culturais em inhame (Colocasia esculenta (L.) Schott) cultivado em solo seco. In: ENCONTRO NACIONAL SOBRE A CULTURA DO INHAME, 2, Dourados. 1989. Anais... Campo Grande: UFMS, 1990b. p. 59-96.

HEREDIA ZÁRATE, N.A. Produção de cinco clones de inhame cultivados no Pantanal SulMatogrossense. Horticultura Brasileira, Brasília, v. 13 , n. 1 , p. $38-40,1995$.
HEREDIA ZÁRATE, N.A.; VIEIRA, M.C., LOPES, J. Produção de rizomas do inhame ' $\mathrm{Cem} /$ Um' sob nove populações de plantas em Dourados-MS. Ciência e Agrotecnologia, Lavras, v. 24, n. 1, p. 118-123, 2000.

HEREDIA ZÁRATE, N.A.; VIEIRA, M.C.; ORTIZ, A.C.S. Produção de clones de cará em função de tipos de mudas. SOBInforma, Rio de Janeiro, v. 17, n. 1, p. 16-17, 1998.

LARCHER, W. Ecofisiologia vegetal. São Carlos: RiMa Artes e Textos. 2000. 531 p.

OLIVEIRA, A.P.; FREITAS NETO, P.A.; SANTOS, E.S. Produtividade do inhame em função de fertilização orgânica e mineral e de épocas de colheita. Horticultura Brasileira, Brasília, v. 19, n. 2, p. 144-147, 2000.

SANTOS, E.S. Inhame (Dioscorea spp.): aspectos básicos da cultura. João Pessoa: EMEPA-PB/ SEBRAE, 1996. 158p.

SANTOS, J.N. Sistema de produção de inhame da Região de Inhapim-MG. In: CORRÊA, L. G. (Coord.). Anais do I Encontro Nacional sobre a cultura do inhame (Colocasia esculenta). Viçosa: UFV, 1994. p. 4-6.

SANTOS, J.N.; CARVALHO, F.A.; CASTILHO, V.H.L.; MELO, M.R.; CORREIA, L.G. A cultura do inhame (Colocasia esculenta Schott). Belo Horizonte, EMATER-MG, 1983. 28 p.

SEDIYAMA, M.A.N.; CASALI, V.W.D. Propagação vegetativa da mandioquinha-salsa. Informe Agropecuário, Belo Horizonte, v. 19, n. 190, p. 24-27, 1997.

VIEIRA, M.C. Avaliação do crescimento e da produção de clones e efeito de resíduo orgânico $e$ de fósforo em mandioquinha-salsa no Estado de Mato Grosso do Sul. Viçosa, 1995. 146 p. (Tese doutorado), UFV.

WANG, J.K. Taro: a review of Colocasia esculenta and its potential. Honolulu: University of Hawaii Press, 1983. 400 p. 